\title{
Editorial: Looking Ahead
}

I am honored to serve Physical Review Letters (PRL) as the Lead Editor. My experience as interim Lead Editor has confirmed that PRL is well run and on track to remain the go-to journal for the physics community. This is the result of the ongoing work of our editors and a legacy of Pierre Meystre's effort as Lead Editor. It is my intention to continue strengthening PRL's role and status in the future.

We cannot be complacent, however, so watch for an upcoming editorial about future initiatives. Already we have asked our board members, the hundred plus Divisional Associate Editors, to be more of an active network in the community-to collect information for the journal, and to disseminate news from the editorial office. But mostly PRL counts on you, readers, authors, and referees. PRL is a community-wide flagship journal not run for profit but for the benefit of all. We welcome your input and your participation in making an even better PRL.

Published 4 April 2017

Hugues Chaté

Editor

American Physical Society

DOI: 10.1103/PhysRevLett.118.140002 